\title{
Two-stage method for the simulation of the comminution process in a high-speed beater mill
}

\author{
Michat J. Marijnissen ${ }^{1 *}$, Cezary Graczykowski ${ }^{1}$, Jerzy Rojek $^{1}$ \\ ${ }^{1}$ Institute of Fundamental Technological Research, Polish Academy of Sciences, Pawińskiego 5b, 02-106, Warsaw, Poland
}

\begin{abstract}
The paper presents a two-stage simplified method for the simulation of comminution process which takes place in a beater mill. The first stage of the proposed method is a simulation of the flow of gas and ore particles through a mill based on a two-phase continuous-discrete model. It allows to capture the interaction between the fluid flow and embedded particles, to determine trajectories of their motion and average velocities and frequencies of their collisions against the flywheel and the mill's walls. The second stage of the proposed method is a discrete element method simulation of the process of comminution of a single ore particle. It allows to determine the size distribution of created smaller particles in terms of normal velocity and angle of impact and to estimate the global efficiency of the comminution process. The proposed simulation methodology is applied for the verification of the innovative concept of the application of high-speed beater mill for the comminution of the copper ore.
\end{abstract}

\section{Introduction}

Beater mills are commonly used in coal power plants for final stage comminution of coal before the combustion stage. Their operation is based on a spinning flywheel, which induces the gas flow and allows the incoming coal particles to achieve high velocity. As a result, the particles collide at high speeds against blades of the flywheel and internal walls of the mill and they are effectively crushed into smaller fractions. The process of high-speed comminution conducted in beater mills is characterised by high efficiency and relatively small energy consumption in comparison to other competitive methods.

The process of comminution in a beater mill is one of the most difficult industrial processes for numerical simulations. The reason for this is the multiplicity and complexity of diverse physical phenomena occurring simultaneously inside the mill during its operation. Amongst them, we can distinguish highly turbulent flow of the gas in the vicinity of the flywheel, the interaction of the flow and particles and, eventually, the ubiquitous process of particles comminution. All these phenomena are mutually dependent and they influence each other in an intrinsic way. Moreover, they all substantially contribute to mill operation and to the efficiency of the comminution process.

The above factors cause that direct numerical simulations of the comminution process inside beater mills are beyond the range of present computational capabilities. The literature on the holistic numerical simulation of the comminution in such a mill is rather limited. Nevertheless, various theoretical approaches and numerical models covering selected aspects of beater mill operation are extensively developed.

The first group of methods is dedicated to the precise simulation of the flow including solid particles with the use of two-phase models. This approach is based on a continuous-discrete Computational Fluid Dynamics Discrete Element Method (CFD-DEM) coupling, which has been used in simulations and validations of particulate flow in a channel [1], fluidised beds [2] and slurry flow [3]. Additionally, it has successfully been tested against analytic and experimental data [4].

The second group of methods is dedicated to the simulation of the comminution process itself. Among these methods, the Discrete Element Method has gained the largest popularity and has been applied to the largest range of problems. The applications of DEM include impact breakage of particle agglomerates [5], impact crushing of particle-particle compounds [6] and ore breakage in laboratory scale ball mill [7]. Moreover, the mechanisms of impact fragmentation were studied in [8], the process of fragmentation in geomaterials was analysed in [9], while ultrasound assisted particle fragmentation was investigated in [10]. An extensive review of the application of DEM for the processes of fragmentation and comminution is presented in [11].

The objective of this paper is a joint application of the methods from two above groups for comprehensive simulation of the process of comminution in a beater mill. The proposed approach consists of two separate stages. The first step is a simulation of the flow of a mixture of gas and ore particles through a beater mill conducted by using two-phase continuous-discrete CFD-DEM model. The conducted simulation enables to observe the

$\overline{\text { *Corresponding author: mmarijn@ippt.pan.pl }}$ 
interaction between the fluid flow and particles, determining their trajectories of motion and collision velocities. This data will be effectively used in the second stage of the method which is a DEM simulation of the comminution of a single ore particle. The conducted DEM simulation will allow to determine the mass distribution of created smaller particles in terms of normal impact velocity and to estimate the global efficiency of the comminution process. The entire proposed simulation methodology will provide deep insight into the process of comminution in beater mills. In particular, it will be applied to the verification of the innovative concept of the application of high-speed beater mills for the comminution of copper ore.

\section{Models used in the computation of the flow in a beater mill}

In the model used the fluid is considered a continuum, however the particles are discrete and tracked in a Langrangian reference frame. The fluid was considered compressible and the flow turbulent. The $\mathrm{k}-\omega$ turbulence model was used to take into account for turbulent flow. Mass and heat exchange between phases were omitted.

\subsection{Fluid flow}

The Finite Volume Method is used to calculate the fluid flow taking into consideration the granular phase's volume fraction in given cells. A multiphase fluid is modelled with the use of conservation equations for a particular phase as given by

$$
\begin{gathered}
\frac{\partial \rho}{\partial t}+\nabla \cdot\{\rho \boldsymbol{u}\}=0 \\
\frac{\partial \rho \boldsymbol{u}}{\partial t}+\{\boldsymbol{u} \cdot \nabla\} \boldsymbol{u}=\rho \boldsymbol{g}-\nabla p_{r}+\nabla \cdot \overline{\tau_{q}} \\
\overline{\tau_{q}}=\mu\left\{\nabla \mathbf{u}+\nabla \mathbf{u}^{T}\right\}+\left\{\lambda_{\mathrm{q}}-\frac{2}{3} \mu\right\} \nabla \cdot \boldsymbol{u} \mathrm{I} \\
\frac{\partial h \rho}{\partial t}+\nabla \cdot\{\rho \boldsymbol{u} h\}=\frac{\partial p_{r}}{\partial t}+\overline{\tau_{q}} \cdot \nabla \boldsymbol{u} .
\end{gathered}
$$

In these equations $\boldsymbol{u}$ is the velocity of the fluid, $\boldsymbol{g}$ the gravity, $\rho$ the density, I the indentity matrix and $p_{r}$ the pressure. Additionally, $\lambda_{q}$ and $\mu$ denote the shear and bulk viscosity, $\overline{\tau_{q}}$ is the stress tensor and $h$ is the specific enthalpy, while $t$ is the time variable. Details of the k- $\omega$ turbulence model can be found in [12].

\subsection{Discrete Element Method}

The DEM was first introduced in [13]. In the DEM we track the position of discrete particles immersed in the fluid as given by

$$
\begin{gathered}
\frac{d \boldsymbol{u}_{s}}{d t}=F_{\text {drag }}\left(\boldsymbol{u}-\boldsymbol{u}_{\boldsymbol{s}}\right)+\frac{\boldsymbol{g}\left\{\rho_{s}-\boldsymbol{\rho}\right\}}{\rho_{s}}+\boldsymbol{F}_{\boldsymbol{a d d} .}, \\
F_{\text {drag }}=\frac{3 \mu C_{d} R e}{4 \rho_{s} \mathrm{~d}_{\mathrm{s}}^{2}}
\end{gathered}
$$

$$
\begin{gathered}
R e=\frac{\rho \mathrm{d}_{\mathrm{s}}\left|\boldsymbol{u}_{s}-\boldsymbol{u}\right|}{\mu}, \\
C_{d}=\left\{\begin{array}{cl}
0.424 & : R e>1000 \\
\frac{24}{R e}\left\{1+\frac{R e^{2 / 3}}{6}\right\} & : R e \leq 1000
\end{array} .\right.
\end{gathered}
$$

Here equation (5) represents the equilibrium of forces acting upon the particles. All variables containing the subscript $s$ consider the particles. Moreover $\boldsymbol{F}_{\text {add }}$. represents additional accelerations acting upon the particle (ex. contact, coupling, pressure gradient) and $F_{\text {drag }}$ can be described as shown in equations (6) and (7). Lastly remains the definition of the particle drag coefficient. We did not take into account other particles shapes than spherical, so we used the formula presented by equation (8) without any shape coefficients.

\subsection{Particle contact}

Contact between particles is considered. The particles are allowed to overlap for a small distance. This overlap is the deciding factor while calculating the contact force. For the overlap to be small it is crucial to use a small time step. When an overlap is detected contact forces are calculated and applied to the particles. The governing equations are given by

$$
\begin{gathered}
\boldsymbol{e}_{12}=\frac{\boldsymbol{x}_{2}-\boldsymbol{x}_{1}}{\left\|\boldsymbol{x}_{2}-\boldsymbol{x}_{1}\right\|}, \\
\delta=\left\|\boldsymbol{x}_{2}-\boldsymbol{x}_{1}\right\|-\left\{r_{2}+r_{1}\right\}, \\
\boldsymbol{F}_{1}=\boldsymbol{F}_{2}=\left\{\mathrm{k} \delta+\gamma \boldsymbol{u}_{12} \cdot \boldsymbol{e}_{12}\right\} \boldsymbol{e}_{12}, \\
\boldsymbol{F}_{\text {tangential }}=\beta \boldsymbol{F}_{1} .
\end{gathered}
$$

Here $\boldsymbol{x}$ is the particle position, $\boldsymbol{r}$ is the radius of the particle, $\mathbf{k}$ and $\boldsymbol{\gamma}$ are the spring and dampening coefficients and $\boldsymbol{\beta}$ is the friction coefficient.

\subsection{Coupling}

Due to low particle concentration, the effect of their presence in the fluid was omitted, creating a 1-way coupling. The fluid's velocity is calculated at the particle's position and passed to equation (5). Next, the particles positions are updated after which the calculations continue for the fluid.

\section{The first stage - fluid and particle flow}

\subsection{Geometry, mesh and boundary conditions}

The simplified geometry of an existing beater mill was used for the simulation domain. The height of the mill was around $4 \mathrm{~m}$, with a flywheel of approximately $1 \mathrm{~m}$ in diameter. The geometry is shown in Fig. 1. with inlets and outlets marked with coloured arrows. The mesh consists of approx. 600.000 finite volumes. A pressure inlet and outlet boundary condition were imposed on the marked surfaces. The flywheel and its nearest vicinity was set in 
a rotary frame and connected with the rest of the mill with an interface boundary condition. The flywheel had a constant rotational velocity of $2000 \mathrm{rpm}$. The fluid time step was set to $5 \cdot 10^{-5} \mathrm{~s}$, while the particle time step was $5 \cdot 10^{-6} \mathrm{~s}$. Such a small time step was required to stabilise the simulation caused by the small gap between the rotary frame and the stationary mesh near the wall.
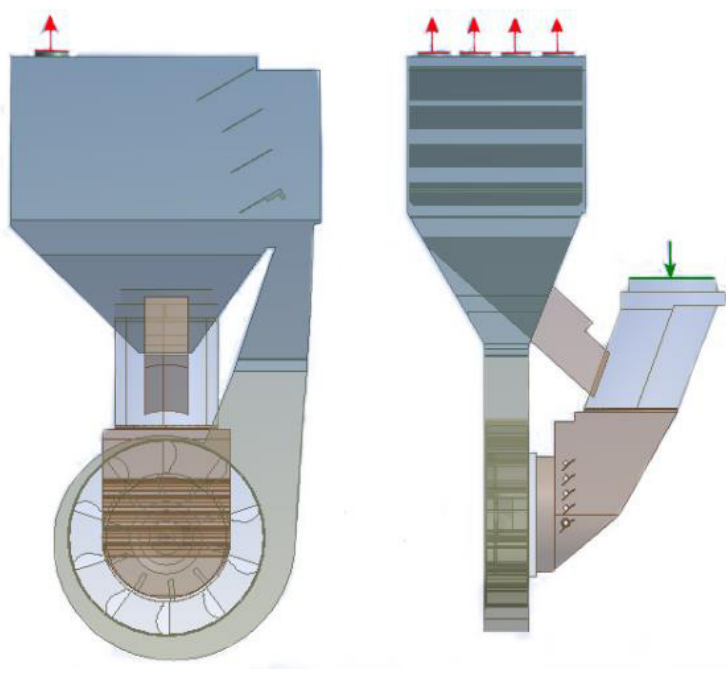

Fig. 1. Geometry of the mill, from the back (left) and side (right). Marked inlet and outlets.

\subsection{Results obtained}

Result obtained with the continuous-discrete method allow for detailed insight in the trajectory of every single particle. Additionally, particles of different size, density and shape can be injected inside the domain. In these calculations, we limited ourselves to spherical sandstone particles with a density of $2580 \mathrm{~kg} / \mathrm{m}^{3}$, based on [14]. Due to a heavily increasing computation cost with the number of particles tracked, we used a group of 360 particles of a given diameter of $40 \mathrm{~mm}$. The group was evenly distributed on the inlet boundary and injected into the domain with a velocity similar to the fluid. The injection was done after the fluid flow inside the mill stabilised. The analysis of particles velocities amongst their trajectories provided information about the impact velocity and angles that occurred during collisions with the flywheel. The results for a single particle are presented in Fig.2, where the collision with the flywheel occurred at approx. $0.18 \mathrm{~s}$.

The results obtained from the group of particles gave a good insight into the impact statistics. The mean velocity of impact was $131.6 \mathrm{~m} / \mathrm{s}$ with a variance of $17.2 \mathrm{~m} / \mathrm{s}$. An image of the particles positions just after the first collisions with the flywheel occurred was captured and is presented in Fig. 3. The image shows the chaotic trajectories after the collision with the flywheel. Particles collide not only with the wall because some of them are reflected back upon the flywheel. This leads to the conclusion that, even if the particles wouldn't break at the first collision the subsequential collision would make sure the particle would crumble. Due to the drastic differences in the behaviour of pre- and post-comminution particles further calculations of the simulation presented in Fig. 3 have not been carried out.

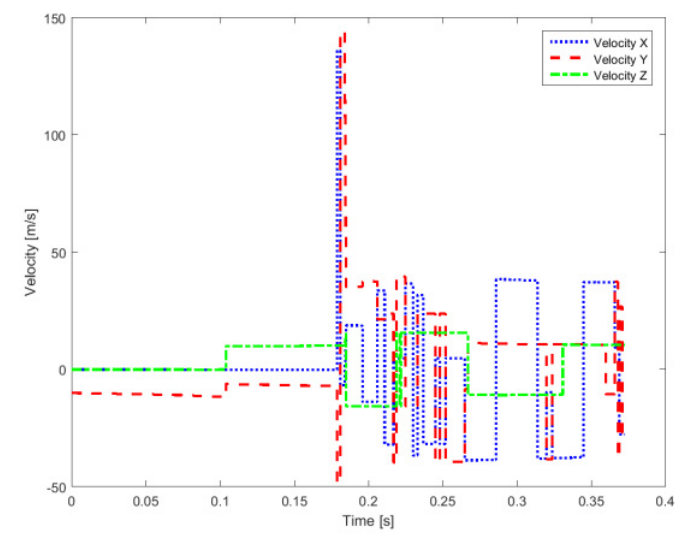

Fig. 2. The velocity of a single particle in a function of time during its transit.
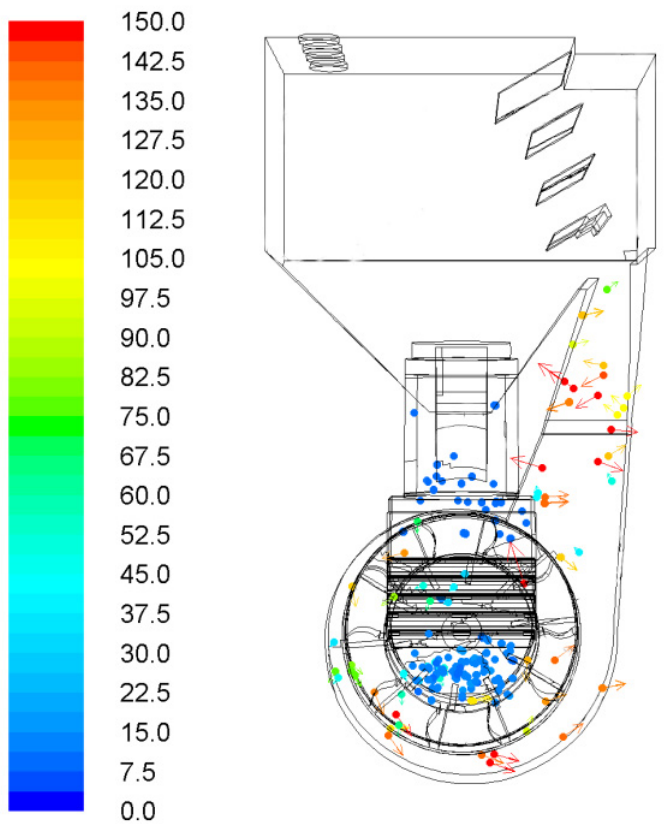

Fig. 3. Particle position in the mill after 0.2039 seconds upon injection coloured by their velocity magnitude $[\mathrm{m} / \mathrm{s}]$. Particle diameter is $40 \mathrm{~mm}$.

For the simulation to have relevance with the existing counterpart, we have restarted the simulation with particles of sizes that can be found in the mill's output. For a detailed analysis of beater mills with this method, we refer the reader to [15].

\section{The second stage - DEM simulation of the elementary comminution process}

The elementary process of comminution is the process of crushing a single material particle during impact against a rigid surface [16]. Mechanical response of the particle during such event strongly depends on its mechanical 
properties including viscoelastic or viscoplastic characteristics and fracture durability, as well as on impact parameters such as velocity, angle and particle orientation.

The process of comminution is especially sensitive to impact velocity. Small impact velocities cause generation of micro-damages and micro-cracks which are often not visible at the particle's surface. The increase of impact velocity results in the generation of more severe fractures and local fragmentation of the particle in the vicinity of the contact area. Eventually, high impact velocities exceeding the critical value cause breakage of the entire particle into numerous pieces of diverse shapes and sizes, and generation of various material fractions

Conducting an elementary test of comminution is crucial for determining the crushing durability of the material. In particular, it enables to define the requirements for the industrial processes of crushing and grinding, to assess their efficiency and to predict the required energy consumption.

The proposed method of a comminution process simulation is based on the classical formulation of the Discrete Element Method [17,18]. In this method, the crushed material particle is modelled by a set of discrete elements, usually spheres of various sizes. At the initial state, the discrete elements are connected with each other by predefined cohesive bonds, providing the initial shape and geometrical configuration of the particle. The impact of this particle against a rigid surface causes breakage of selected cohesive bonds and resulting disintegration of the modelled ore particle into a set of smaller pieces, Fig. 4.

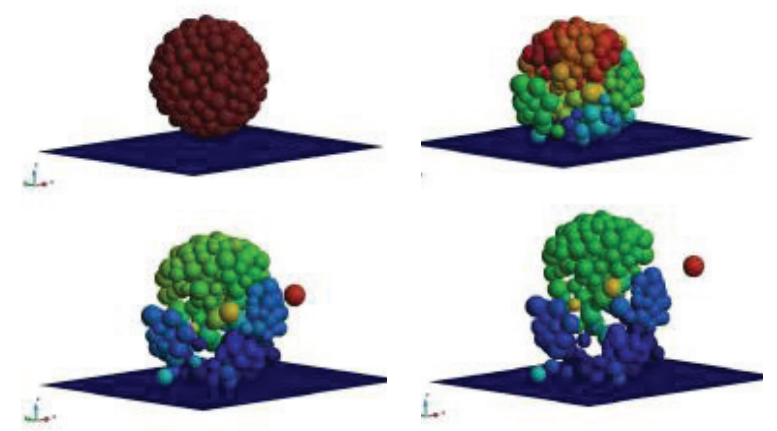

Fig. 4. Numerical simulation of the comminution of a single material particle by Discrete Element Method [18].

The mathematical formulation of the DEM model is based on equations describing the motion of each particle, which include inertial and contact forces. The inertial forces are expressed by the second derivative of the particle displacement vector $\mathbf{u}$ defined in a classical Lagrange reference frame. In turn, the contact forces transferred to the particles can be replaced by the point load $\mathbf{F}^{\text {ext }}$ and concentrated torque $\mathbf{T}^{\mathbf{e x t}}$ applied at the centre of inertia of each particle. Consequently, the equations of motion of a single particle read:

$$
m \ddot{\boldsymbol{u}}_{C}=\boldsymbol{F}^{\boldsymbol{e x t}}, J_{C} \dot{\boldsymbol{\omega}}=\boldsymbol{T}_{C}^{\boldsymbol{e x t}},
$$

while matrix equilibrium equations of the whole system of particles assume the form:

$$
M r=R, J \dot{\Omega}=T,
$$

where $\mathbf{M}$ and $\mathbf{J}$ are the matrices containing masses and moments of inertia of the discrete elements, whereas vectors $\mathbf{R}$ and $\mathbf{T}$ contain point loads and concentrated torques acting on particles. The above equations are solved by using an explicit method of integration.

The contact interactions acting between the discrete elements can be decomposed into a direction normal and tangential to the surface of contact. The contact force generated in the normal direction is defined by stiffness and damping coefficients, while the contact force generated in the tangential direction is defined by the stiffness and friction coefficients, Fig. 5.
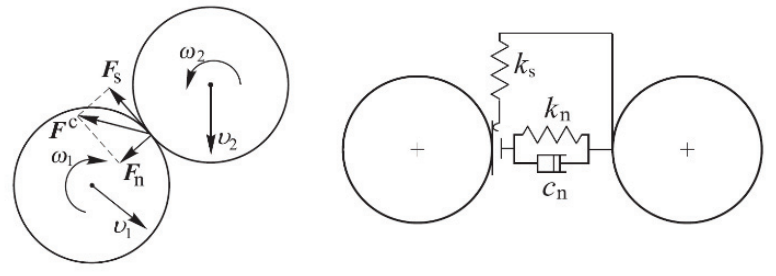

Fig. 5. Contact interactions between the discrete elements [17].

Accordingly, the cohesive bonds providing the possibility of solid material modelling are defined separately in the normal and tangential direction to the contact surface. Finally, the possibility of breakage of the cohesive bonds is provided by using stress-based criteria of breakage depicted in Fig. 6.

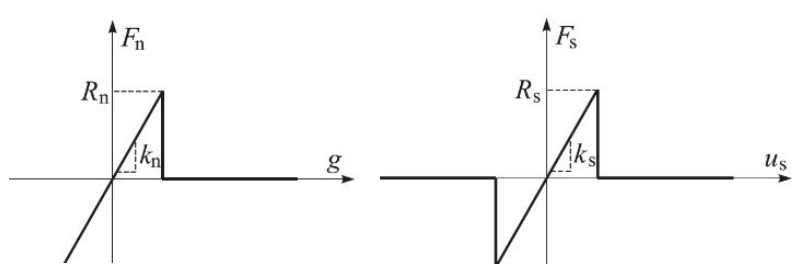

Fig. 6. Applied model of cohesive bonds with breakage [17].

All results of the elementary comminution process presented in this paper were obtained by the use of the discrete element method - based software DEM-Pack developed by the third author [17].

\section{Results of DEM simulations of the comminution process}

\subsection{Model description}

The simulations of the elementary comminution process were performed for an idealised particle of spherical shape with a diameter equal to $40 \mathrm{~mm}$. The analysed model is composed of 3344 spherical elements with various radii of an average size of $1,3 \mathrm{~mm}$. The average filling of the model by solid material is approximately $92 \%$. The defined initial cohesive bonds provide that their 
average number corresponding to a single element equals approximately six.

The model includes a flat surface located in the vicinity of the particle, which models the rigid obstacle as well as the definition of the contact conditions between the particle and rigid surface. The initial velocity applied to the particle causes its rapid contact with the surface resulting in breaking the subsequent contact bonds and initiation of the comminution process.

The conducted numerical studies were aimed at the investigation of sandstone, being the carrier of copper ore. The macro-mechanical parameters of the sandstone were assumed as equal to: $\rho=2580 \mathrm{~kg} / \mathrm{m}^{3}, \mathrm{E}=18.69 \mathrm{GPa}, v=$ $0.18, \sigma_{\mathrm{c}}=50 \mathrm{MPa}, \sigma_{\mathrm{t}}=5 \mathrm{MPa}$, based on [14]. Further, the micro-mechanical parameters of DEM model were determined by using the dependences defined in [17]. The micro-mechanical parameters were assumed to be equal for the entire comminuted particle. The possible inhomogeneity resulting from the mineralogical composition of the material was not taken into account at this stage of research.

\subsection{Elementary low-speed comminution process of sandstone}

The initial numerical test was a simulation of the elementary process of comminution of a sandstone particle at relatively low impact velocity of $37 \mathrm{~m} / \mathrm{s}$. The applied velocity was sufficient to cause breakage of the particle into 925 separate pieces, including 826 totally shredded particles (containing only one discrete element) and 99 partially shredded particles (containing more than one discrete element).

The initial configuration of the particle, including colouring indicating final division into pieces is presented in Fig. 7a, while the configuration of the system occurring $6 \mathrm{~ms}$ after the beginning of impact is presented in Fig. 7b (partially shredded particles are marked in various colours, while totally shredded particles are marked in red).

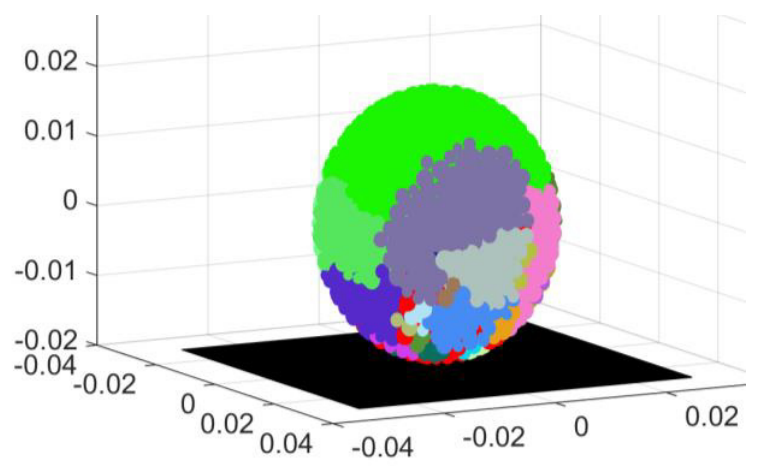

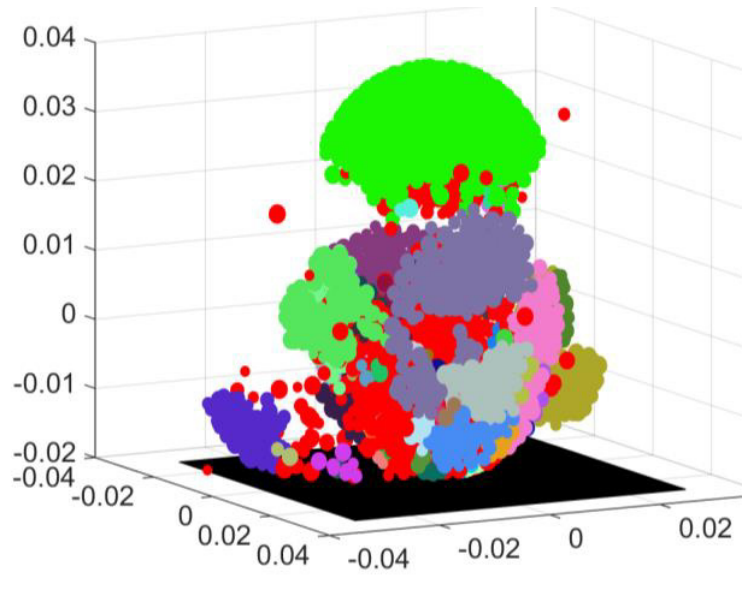

Fig. 7. Results of the numerical simulation of the elementary comminution process of a sandstone particle (impact velocity equal $37 \mathrm{~m} / \mathrm{s}$ ) : a) the initial state, b) state $6 \mathrm{~ms}$ after beginning of impact.

\subsection{Sensitivity of the process of comminution on the initial impact velocity}

The following objective was an investigation of impact velocity influence on the elementary process of comminution. In order to avoid a detailed analysis of the grain size distribution for each particular impact velocity, two global coefficients summarising the process of comminution were introduced:

- the number of partially shredded particles,

- the size of the largest particle.

Since both coefficients describes different properties of particle breakage they can be considered as a good global measure of the effectiveness of the comminution process. In the presented example, the comminution of a sandstone particle was analysed in a wide range of velocities from 4 to $169 \mathrm{~m} / \mathrm{s}$. Results of the conducted sensitivity study are shown in Fig. 8a-b.

The obtained results allow to assessquantitatively the intuitive effect of the intensification of the comminution process caused by an increase of the impact velocity. The critical velocity, which can be distinguished from the plot of partially shredded particles (Fig. 8a) and the plot of largest particle size (Fig. 8b) equals approximately 70 $\mathrm{m} / \mathrm{s}$. For this velocity, the particles which previously were only partially shredded become broken into smaller pieces, while the size of the largest particle decreases below $4 \%$ of the original particle size. Simultaneously, the number of fully shredded particles exceeds 2000 which allows to consider the analysed single impact comminution process as very efficient. 

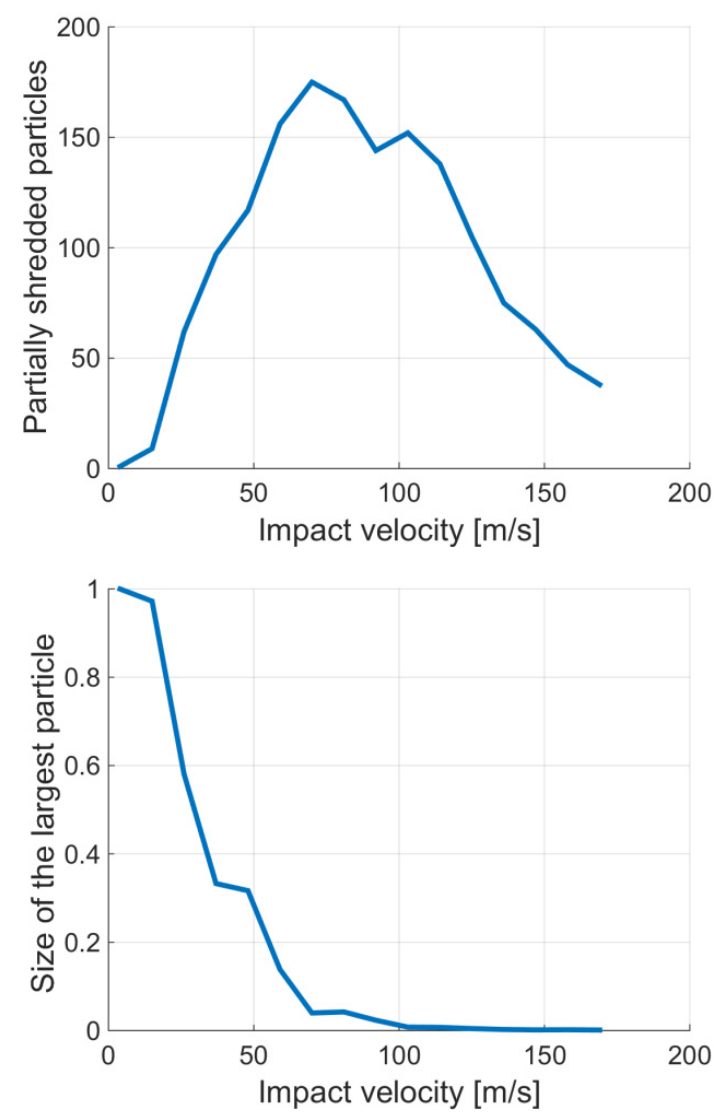

Fig. 8. Sensitivity of the sandstone particle comminution on impact velocity in the range $4-169 \mathrm{~m} / \mathrm{s}$ : a) number of fully shredded particles, b) relative size of the largest particle.

\subsection{High-speed comminution in a beater mill at $2000 \mathrm{rpm}$}

The final analysis was simulation of the comminution of a sandstone particle under conditions which occur inside the beater mill operating at rotational velocity of 2000 rpm. The impact velocities in the corresponding DEM simulations covered the range 125-169 $\mathrm{m} / \mathrm{s}$. Five simulations of the comminution of spherical sandstone particle of diameter $40 \mathrm{~mm}$ were conducted and their results were collected together in order to find averaged characteristics of the process, Fig. 9.

The obtained results indicate a very high average level of comminution within the considered range of impact velocities. The products of the comminution are grouped in 12 fractions, among which the largest has the size of $0.45 \%$ of the original particle. Almost $94.6 \%$ of the total mass of the product are fully shredded elements, which belong to the fraction $0-0.03 \%$ (the entire vertical range is not shown in Fig. 9 for better visibility). Moreover, the particles smaller than $0.12 \%$ of the original size constitute almost $99 \%$ of total mass of the product.

All the above-described results indicate that the assumed range of the flywheel's rotational velocity of $2000 \mathrm{rpm}$ is definitely sufficient for the purpose of sandstone comminution. Taking into account the occurrence of multiple collisions inside the mill, the assumed velocity can be regarded as too large and its decrease should be considered.

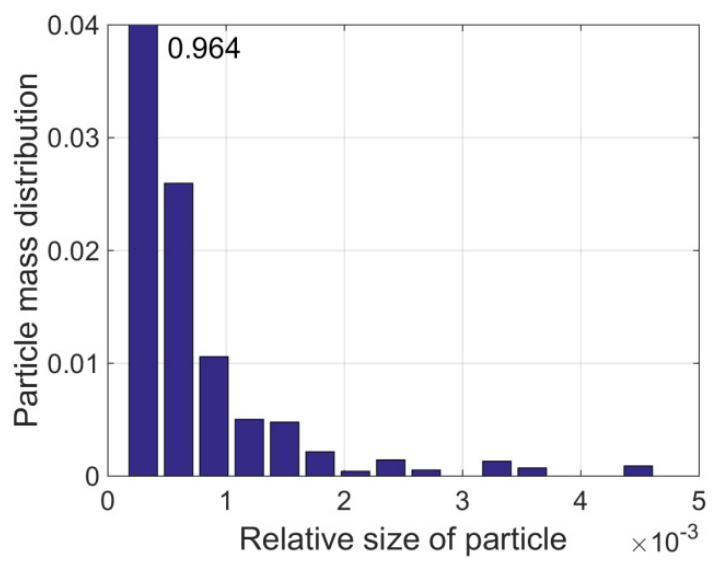

Fig. 9. Relative mass distribution resulting from comminution of a sandstone (impact velocity $125-169 \mathrm{~m} / \mathrm{s}$ ).

\section{Conclusions}

The paper introduces a simplified method for the numerical analysis of the comminution process inside a beater mill. It includes simulations of the flow of the mixture of gas and solid particles through the mill by using continuous-discrete CFD-DEM model and the following simulations of the elementary process of comminution by using the DEM model including bonds breakage.

Although the proposed method does not provide full interaction between the flow and the particle's breakage it allows to preliminarily investigate the effectiveness of the conducted comminution's process. In particular, the method was successfully applied for the verification of the innovative concept of applying beater mills for the comminution of sandstone and the extraction of copper ore. It was proved that the applied rotational velocity of the flywheel of $2000 \mathrm{rpm}$ allows to achieve more than satisfactory results of sandstone comminution.

\section{References}

1. M. Azimian, M.Lichti, H.J. Bart, Open Chem. Eng. J.8, 1-11, (2014)

2. C. Yang, Y. Duan, Chem. Eng. Technol. 36, 19071914 (2013)

3. D. Kubicki, S. LO,Ninth International Conference on CFD in the Minerals and Process Industries.1012December (2012)

4. C. Kloss et al., Progr. Comp.Fluid Dyn. 12,140-152 (2012)

5. B.K. Mishra, C. Thornton, Int. J. Miner. Process. 61, 225-239 (2001)

6. W. Schubert, M. Khanal, J. Tomas, Int. J. Miner. Process. 75, 41- 52(2005)

7. P.W. Cleary, R.D. Morrison, Miner. Eng. 24, 352366(2011) 
8. F. K. Wittem et al., Int. J.Fract. 154, 105-117 (2008)

9. G.A. D'Addettaet al., Lect. Not. Phys., 568, 231-258 (2001)

10. F. K. Wittel, Gran. Matt. 12,447-455 (2010)

11. H.A. Carmona, F.K. Wittel, F.Kun,Eur. Phys. J. Special Topics 223, 2369-2382 (2014)

12. D.C. Wilcox, DCW Indust. Inc. La Canada, (1998)

13. P.A. Cundall, O.D.Stark, Géotechnique, 29, 4765 (1979)

14. N. Kunysz, Rudy i Metale, (1972)
15. M.J. Marijnissen, J. Rojek, J. Phys. Conf. Ser. 760, 18 (2016)

16. M.Brozek,W. Maczka,T. Tumidajski, Modele matematyczne procesów rozdrabniania (1995)

17. J.Rojek, Modelowanie i symulacja komputerowa zlozonych zagadnien mechaniki nieliniowej metodami elementów skonczonych $i$ dyskretnych (2007)

18. J.Rojek, C.Graczykowski, M.J.Marijnissen, T.Szolc, I.Marczewska, Proceedings of the XIth International Conference on Non-ferrous Ore Processing (2015) 